\title{
Interaction between TCF7L2 rs7903146 Genotype, HbA1c Levels, and the Periodontal Status of Dental Patients
}

\author{
Andreas Grigoriadis ${ }^{1,2}$ \\ Symela Koutounidou ${ }^{3}$ \\ ${ }^{1}$ Department of Preventive Dentistry, Periodontology and Implant \\ Biology, Dental School, Aristotle University of Thessaloniki, \\ Thessaloniki, Greece \\ 2Department of Periodontology, 424 General Military Training \\ Hospital, Thessaloniki, Greece \\ ${ }^{3}$ Department of Genetics and Molecular Biology, School of Biology, \\ Aristotle University of Thessaloniki, Thessaloniki, Greece \\ ${ }^{4}$ Department of Oral and Maxillofacial Diseases, Helsinki University \\ and University Hospital, Helsinki, Finland
}

Eur J Dent 2021;15:495-501

\author{
Address for correspondence Andreas Grigoriadis, Department \\ of Periodontology, 424 General Military Training Hospital, 54621 \\ Thessaloniki, Greece (e-mail: andreasgrigor@gmail.com).
}

\section{Abstract}

Keywords

- genotype

- HbA1c

- prediabetes

- periodontitis
Objective The aim of the study was to investigate the potential interaction between TCF7L2 rs7903146 genotype, which is implicated for type-2 diabetes mellitus genetic susceptibility, HbA1c levels, and the periodontal status of dental patients.

Materials and Methods HbA1c levels, clinical periodontal parameters (probing depth, clinical attachment level, bleeding on probing, and plaque index), and several parameters (such as body mass index [BMI], smoking habits, education level, and age) were recorded in 150 patients who fulfilled the criteria for screening for prediabetes/diabetes of the Centers for Disease Control and Prevention. DNA was extracted and the TCF7L2 single nucleotide polymorphism (SNP) rs7903146 was genotyped in all participants.

Results Thirty-one patients out of 150 tested were found with unknown hyperglycemia (20.7\%). Regarding sex, education, parent with diabetes, normal BMI, smoking, age $\geq 45$ years and prior testing for diabetes, no differences were observed between patients displaying HbA1c $<5.7$ and $\geq 5.7 \%$ (Pearson's Chi-square test, $p>0.05$ ). Regarding periodontal parameters and differences between subgroups (HbA1c levels $\geq 5.7$ and HbA1c levels $<5.7$ ), statistically significant differences were observed for probing depth (3.20 \pm 0.94 vs. $2.81 \pm 0.78 \mathrm{~mm}$ ), clinical attachment level ( $3.54 \pm 1.20$ vs. $3.18 \pm 1.06 \mathrm{~mm}$ ) and bleeding on probing $(0.62 \pm 0.25$ vs. $0.50 \pm 0.24 \%)$ with hyperglycemic patients exhibiting worse periodontal conditions (Mann-Whitney test $p<0.05$ ). The allelic and genotype frequencies for the transcription factor 7-like 2 (TCF7L2) gene, SNPs 7903146 did not exhibit a significant difference between the HbA1c $>5.7$ and HbA1c $<5.7$ groups and the periodontitis and nonperiodontitis subgroups respectively (Fisher's exact test $>0.05$ ). Statistical Analysis Patient characteristics and their association with prediabetes were tested by Pearson's Chi-square test (asymptotic, two sided). Differences of periodontal parameters between subgroups were tested with the Mann-Whitney U-test. The associations of allele and genotype frequencies in the patient and control groups were analyzed using the Fisher's exact test of independence.The significance level was set at the 0.05 for all tests.

Conclusion A statistically significant association between TCF7L2 rs7903146 genotype and periodontal condition or HbA1c levels was not observed in contrast to statistically significant differences of clinical parameters of periodontitis in patients with hyperglycemia. published online May 26, 2021
DOI https://doi.org/

$10.1055 / \mathrm{s}-0041-1725578$ ISSN 1305-7456.
(C) 2021. European Journal of Dentistry.

This is an open access article published by Thieme under the terms of the Creative Commons Attribution-NonDerivative-NonCommercial-License, permitting copying and reproduction so long as the original work is given appropriate credit. Contents may not be used for commercial purposes, or adapted, remixed, transformed or built upon. (https://creativecommons.org/licenses/by-nc-nd/4.0/).

Thieme Medical and Scientific Publishers Pvt. Ltd. A-12, 2nd Floor, Sector 2, Noida-201301 UP, India 


\section{Introduction}

Periodontal disease in the forms of gingivitis and periodontitis is widespread and significantly affecting both individual welfare and health care systems. ${ }^{1}$ While extensive epidemiological data ${ }^{2}$ has shown that periodontal disease increases the risk of poor glycemic control in patients diagnosed with type-2 diabetes mellitus (T2DM), as well as diabetes complications and associated morbidity, it has also been proven that prediabetes and diabetes are associated with an increased rate of initiation, progression and severity of periodontal disease in the oral cavity so oral health professionals are strongly recommended to contribute to the early diagnosis of these conditions in dental patients.

In fact, a number of studies ${ }^{3,4}$ has shown that screening for prediabetes/diabetes and referring to physicians, especially when patients fulfill validated questionnaire criteria, is feasible at the dental clinics, albeit differences in the investigated population (patients with periodontal disease or unknown periodontal condition) and the methodology applied (e.g., testing for free glucose levels or HbA1c).

In addition, important information regarding pathogenetic pathways of periodontal disease and T2DM could be provided by investigating inflammatory mediators in serum and/or saliva in order to establish the periodontal systemic interaction as already shown, especially in cardiovascular disease (CVD) and specific biomarkers and immunological response.-7 In particular saliva, due to noninvasiveness, ease in sample collection and handling at the dental settings appears as a feasible tool for screening for both oral and nonoral diseases. ${ }^{8}$

Besides, other than obesity, ethnicity, nutritional, and various lifestyle habits, ${ }^{9}$ T2DM has a strong genetic basis and therefore genetic-based tests could contribute to early diagnosis and treatment. ${ }^{10}$ It is estimated that more than 200 genetic loci have been identified aiming to have the genetic component of T2DM defined. ${ }^{11}$ Among them, polymorphisms of the transcription factor 7-like 2 (TCF7L2) gene which is located on chromosome 10q.25.2-25.3 have been widely identified in populations of different origins. ${ }^{12}$ This protein, part of the Wnt-signaling pathway, is known to regulate basic functions of pancreatic islet cells and also control the transcription of the proglucagongene. ${ }^{13}$ The TCF7L2 gene is not exclusively expressed in pancreas, as it has also been identified in other organs which participate in metabolic homeostasis 9 (liver, gut and skeletal muscle).

An association between this gene and T2DM both overall and in Caucasians, is revealed through original research papers along with meta-analyses showing strong evidence for North Europeans, East Asians, and Indians. More specifically, potential multiplicative genetic model for four single nucleotide polymorphisms (SNPs) of the TCF7L2 gene (rs7901695 T-to-C, rs11196205 G-to-C, rs12255372 G-to-T, and rs7903146C-to-T) among different ethnic populations is suggested allowing for the last two variants to be taken as reference loci in order to explore T2DM susceptibility. ${ }^{12,14}$

The aim of present study was to screen dental patients of Greek origin for prediabetes/diabetes and identify the possible relation of TCF7L2 rs7903146 genotype with this condition and their periodontal status.

\section{Materials and Methods}

\section{Patient Sample}

The sample pool of possible participants consisted of 731 consecutive patients who visited the Department of Periodontology, Dental School, Aristotle University, Thessaloniki, Greece, and the Periodontal Department of 424 General Army Hospital, Thessaloniki, Greece. Patients were deemed eligible as long as they fitted the following inclusion criteria: age $>30$ years, absence of systemic diseases or medications known to affect periodontal tissues, without infectious conditions (hepatitis or HIV), or pregnancy and lactation, who did not receive periodontal treatment or antibiotics within the last 6 months. After applying the self-assessed questionnaire proposed by the Centers for Disease Control and Prevention (CDC, United States), ${ }^{15} 150$ patients being at high risk for developing diabetes mellitus (score $>9$ ) were identified and included in the study. The minimally required sample size for identifying patients with undiagnosed diabetes ( $n=139$ ) was calculated according to the mean percentage of undiagnosed diabetes in Europe (10\%) as described before. ${ }^{16}$ Participants signed an informed consent and the study was conducted according to the protocol outlined by the Research Committee, Aristotle University of Thessaloniki, Greece, and approved by the Ethical Committee of the School of Dentistry (protocol number: 64, 06/12/2018).

\section{Clinical Procedures}

Among patients who fulfilled the CDC criteria, the Cobas b101 (Roche Diagnostics) in vitro diagnostic test system for determination of HbA1c levels was applied. The system determines the amount of HbA1c in human capillary blood by photometric transmission measurement. The method has been standardized against the IFCC (International Federation of Clinical Chemists) reference method. ${ }^{17}$ Several parameters, including body mass index (BMI), age, level of education, and smoking, were also recorded. All patients identified with hyperglycemia $(\mathrm{HbA} 1 \mathrm{c} \geq 5.7 \%)$ were strongly recommended to contact their physician for further consultation, and laboratory tests. Participants were also polled about the ease of the procedure at the dental clinic.

Participants were instructed to provide a $10-\mathrm{mL}$ unstimulated saliva sample in a sterilized plastic centrifuge tubes (Greiner Bio-one International $\mathrm{GmbH}$ ). Prior to the saliva collection, they were instructed to refrain from oral hygiene, eating, drinking, or smoking for at least 1 hour. Saliva samples were kept at $80 \mathrm{C}$ until analyzed. Following saliva collection, a full-mouth periodontal examination was performed with an automated probe (Florida Probe; Florida Probe Corporation, Gainesville, Florida, United States) by one calibrated examiner (A.G.). Periodontal examination included clinical measurement of probing depth (PD), clinical attachment level (CAL), bleeding on probing (BOP), and plaque levels in six surfaces of each tooth, excluding third 
molars. Diagnosis of stage and grade of periodontitis was according to the revised classification of periodontal diseases as described. ${ }^{1}$

\section{DNA Extraction}

DNA was extracted from 150 saliva samples using the Quick-DNA Miniprep Plus Kit (Zymo Research; Irvine, California, United States), following the manufacturer's protocol. The extracted DNA was stored at $-20^{\circ} \mathrm{C}$ until further analysis.

\section{Polymerase Chain Reaction}

Polymerase chain reaction (PCR) for the TCF7L2 SNP rs7903146 was performed using the following primers:

5'-ACAATTAGAGAGCTAAGCACTTTTTAGGTA-3' (forward) and $5^{\prime}$-GTGAAGTGCCCAAGCTTCTC-3' (reverse). ${ }^{18}$

The PCR amplification was carried out in a $25-\mu \mathrm{L}$ reaction volume containing $2 \mu \mathrm{L}$ of template DNA, $0.2 \mu \mathrm{M}$ of each Primer (Integrated DNA Technologies; Coralville, Iowa, United States), $200 \mu \mathrm{M}$ of each dNTP (dNTP Bundle, Jena Bioscience, Jena, Germany), 1X of KAPA Taq Buffer A 10X with $1.5 \mathrm{mMMgCl}_{2}$ (KAPATaq PCR kit, Kapa Biosystems, Inc., Wilmington, Massachusetts, United States; Roche, Basel, Switzerland), 0.5U of KAPA Taq DNA Polymerase (Kapa Biosystems, Inc., Wilmington, Massachusetts, United States; Roche, Basel, Switzerland) and sterile ddH20. The PCR cycling conditions comprised an initial 5-minute heating step at $94^{\circ} \mathrm{C}$, followed by 35 cycles of 30 seconds at $94^{\circ} \mathrm{C}, 30$ seconds at $58^{\circ} \mathrm{C}$, and 30 seconds at $72^{\circ} \mathrm{C}$, and a final extension at $72^{\circ} \mathrm{C}$ for 5 minutes. The PCR products were visualized by $2 \%$ agarose gel electrophoresis using a 100 bp DNA Ladder (Nippon Genetics Europe GmbH, Düren, Germany) and were identified by Midori Green staining (Nippon Genetics Europe GmbH, Düren, Germany).

\section{Restriction Fragment Length Polymorphism}

Genotyping of TCF7L2 polymorphism rs7930146 was carried out by digesting the $188 \mathrm{bp} \mathrm{PCR} \mathrm{products} \mathrm{in} \mathrm{a} \mathrm{reaction}$ mixture with 10U of the restriction enzyme Rsal (Minotech Biotechnology, Heraklion, Crete, Greece), 1X Rsal Buffer (Minotech Biotechnology), and sterile ddH20 up to a final volume of $20 \mu \mathrm{L}$. The reaction mixture was incubated at $37^{\circ} \mathrm{C}$ for 4 hours.

The cleaved products were separated with gel electrophoresis on $2 \%$ agarose gel (-Fig. 1). In the rs7903146 C allele, the Rsal enzyme recognizes the restriction site $5^{\prime}-\mathrm{GT} \mathbf{\nabla} \mathrm{AC}-3^{\prime}$ and produces two fragments, 159 , and $29 \mathrm{bp}$, whereas the rs7903146 T allele is not cleaved, and the PCR product remains $188 \mathrm{bp}$.

\section{Statistical Analysis}

Statistical analyses were performed with the IBM SPSS 25 Statistical Software Package (IBM Corporation, United States). Patient characteristics and their association with prediabetes were tested by Pearson's Chi-square test (asymptotic, two sided) and are displayed in - Table 1. Differences regarding periodontal parameters assessed between subgroups (HbA1c levels $\geq 5.7$ and HbA1c levels $<5.7$ ) were tested with the Mann-Whitney $U$-test. The associations of allele and genotype frequencies in the patient and control groups were analyzed using the Fisher's exact test of independence. Odds ratios (OR) and 95\% confidence intervals (CIs) were also calculated to determine strength of the associations. The significance level was set at the 0.05 for all tests.

\section{Results}

Demographic and anthropocentric characteristics of patients are shown in -Table 1. From the 150 participants, 76 were women and 74 men (average age: 53.3. years). Thirty-one patients out of 150 tested were found to have $\mathrm{HbA} 1 \mathrm{c} \geq$ 5.7\% (20.7\%), 24 of which had HbA1c 5.7 to 6.4\%, indicating undiagnosed prediabetes (16\%), and seven displayed HbA1c $\geq 6.5 \%$ (range: $6.8-7.8 \%$, indicating undiagnosed diabetes (4.7\%). The remaining participants (119 patients) had HbA1c $<5.7$. Regarding variables sex, parent with diabetes, presence of normal BMI, smoking, age $\geq 45$ years and prior testing for diabetes, no statistically significant differences were observed between patients displaying HbA1c levels $<5.7$ and $\geq 5$.7 (Pearson's Chi-square test, $p>0.05$ ).

Regarding periodontal parameters and differences between subgroups (HbA1c levels $\geq 5.7$ and HbA1c levels < 5.7),

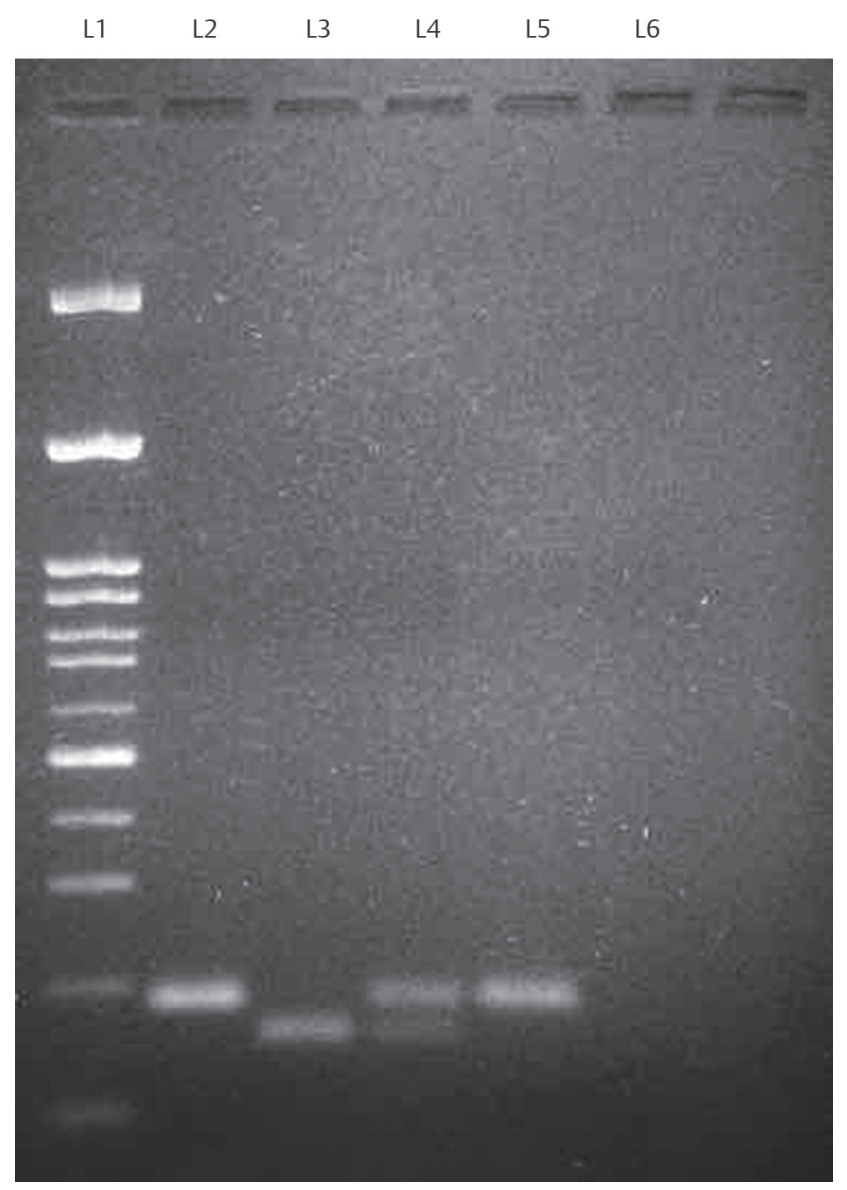

Fig. 1 The 2\% agarose gel electrophoresis of Rsal digested PCR products. Line 1: 100 bp DNA ladder (Nippon Genetics Europe GmbH, Düren, Germany). Line 2: uncleaved PCR product. Line 3: C/C genotype. Line 4: C/T genotype. Line 5: T/T genotype. Line 6: negative control. PCR, polymerase chain reaction. 
Table 1 Demographic and patients characteristics data of participants $(n=150)$

\begin{tabular}{|c|c|c|}
\hline Data & & Mean + SD \\
\hline Age $(y)$ & & $53.33 \pm 11.61$ \\
\hline Weight (kg) & & $88.35 \pm 18.09$ \\
\hline Height (cm) & & $170.74 \pm 9.71$ \\
\hline Waist (cm) & & $102.26 \pm 15.94$ \\
\hline $\mathrm{BMI}\left(\mathrm{kg} / \mathrm{m}^{2}\right)$ & & $30.10 \pm 4.87$ \\
\hline Patients characteristics & & Number (\%) \\
\hline Sex & Women & $76(50.7)$ \\
\hline & Men & $74(49.3)$ \\
\hline Education & Elementary & $3(2.0)$ \\
\hline & Middle & $70(46.7)$ \\
\hline & Technical school & $4(2.7)$ \\
\hline & University & $59(39.3)$ \\
\hline & Postgraduate & $14(9.3)$ \\
\hline Exercise & Yes & $4(2.7)$ \\
\hline Smoking & Yes & $100(66.7)$ \\
\hline & No & $45(30.0)$ \\
\hline & Electronic & $5(3.3)$ \\
\hline Age $\geq 45$ years & Yes & $118(78.7)$ \\
\hline Prior diabetes test & Yes & $123(82.0)$ \\
\hline Annual blood test & Yes & $93(62.0)$ \\
\hline Annual dental visit & Yes & $91(60.7)$ \\
\hline Parent with diabetes & Yes & $54(36.0)$ \\
\hline Brother/sister with diabetes & Yes & $20(13.3)$ \\
\hline Normal BMI & Yes & $40(26.7)$ \\
\hline aMMP-8 & Positive $(\geq 20 \mathrm{ng} / \mathrm{mL}$ ) & $52(34.7)$ \\
\hline HbA1c & $<5.7 \%$ & $119(79.3)$ \\
\hline & $\geq 5.7 \%$ & $31(20.7)$ \\
\hline & $\geq 6.8 \%$ & $7(4.7)$ \\
\hline $\mathrm{HbA} 1 \mathrm{c} \geq 5.7 \%$ & Periodontitis & $29(39.5)$ \\
\hline & Nonperiodontitis & $2(6.5)$ \\
\hline $\mathrm{HbA} 1 \mathrm{c}<5.7 \%$ & Periodontitis & $90(75.6)$ \\
\hline & Nonperiodontitis & $29(93.5)$ \\
\hline
\end{tabular}

Abbreviations: BMI, body mass index; SD, standard deviation.

statistically significant differences were observed for probing depth $(3.20 \pm 0.94$ vs. $2.81 \pm 0.78 \mathrm{~mm})$, clinical attachment level ( $3.54 \pm 1.20$ vs. $3.18 \pm 1.06 \mathrm{~mm}$ ) and bleeding on probing $(0.62 \pm 0.25$ vs. $0.50 \pm 0.24 \%)$ with hyperglycemic patients exhibiting worse periodontal conditions (MannWhitney test $p<0.05)$, but not higher levels of plaque (0.52 \pm 0.27 vs. $0.47 \pm 0.28 \%$ ), respectively (Mann-Whitney test $p$ $>0.05)$. Genotype frequencies for rs7903146 variants $(\mathrm{C} / \mathrm{T})$ in the TGF7L2 gene and distribution of alleles $C$ and $T$ in patients with HbA1c $>5.7$ and $<5.7$ are summarized in - Table 2 . The comparison revealed no association (Pearson's Chi-square test, $p=0.524$ [not significant], Fisher's exact test, $p=0.547$ [not significant]) with HbA1c levels with OR of 0.95 for CC vs (TT and CT) in patients with HbA1c < 5.7 (95\% CI: 0.80-1.12) and 1.23 (95\% CI: 0.66-2.29) in patients with $\geq 5.7 \mathrm{HbA1c}$. Similarly, - Table 3 shows the genotype frequencies and the distribution of alleles $\mathrm{C} / \mathrm{T}$ according to the periodontal condition of the 150 patients. There was no significant difference (Pearson's Chi-square test, $p=0.453$ [not significant], Fisher's exact test $p=0.548$ [not significant]) between carriage of these alleles and the periodontitis and nonperiodontitis subgroups with OR of 1.07 for CC vs (TT and CT) in patients with periodontitis (95\% CI: $0.90-1.26)$ and 0.79 in nonperiodontitis patients (95\% CI: $0.41-1.49)$. 
Table 2 TCF7L2gene rs7903146 genotype frequencies and distribution of alleles C and T in patientswith $\mathrm{HbA} 1 \mathrm{c}>5.7$ and $<5.7$

\begin{tabular}{|c|c|c|c|c|}
\hline & & HbA1c > 5.7 & $\mathrm{HbA} 1 \mathrm{c}<5.7$ & Total \\
\hline \multirow[t]{4}{*}{ TCF7L2 rs7903146 } & & $n=31(\%)$ & $n=119(\%)$ & $n=150(\%)$ \\
\hline & $\mathrm{CC}$ & $15(48.4)$ & $50(42)$ & $65(43.3)$ \\
\hline & CT CC & $12(38.7)$ & $53(44.5)$ & $65(43.3)$ \\
\hline & TT & 4 (12.9) & $16(13.4)$ & $20(13.3)$ \\
\hline \multirow[t]{3}{*}{ TCF7L2 rs7903146 } & & $n=62(\%)$ & $n=238(\%)$ & $n=300(\%)$ \\
\hline & C & $42(67.7)$ & $153(64.3)$ & $238(79.3)$ \\
\hline & $\mathrm{T}$ & $20(32.3)$ & $85(35.7)$ & $62(20.7)$ \\
\hline
\end{tabular}

Note: Pearson's Chi-square test, $p=0.524$ (not significant); Fisher's exact test, $p=0.547$ (not significant), Odds ratio (OR) $=0.95$ for CC vs. (TT and CT) in patients with $\mathrm{HbA} 1 \mathrm{c}<5.7,95 \%$ confidence interval $(\mathrm{Cl}): 0.80-1.12$ and in patients with $\mathrm{HbA} 1 \mathrm{c}>5.70, \mathrm{OR}=1.23 ; 95 \% \mathrm{Cl}: 0.66-2.29$.

Table 3 TCF7L2gene rs7903146 genotype frequencies and distribution of alleles C and T according to periodontal condition

\begin{tabular}{|l|l|l|l|l|}
\hline & & Nonperiodontitis & Periodontitis & Total \\
\hline TCF7L2 rs7903146 & & $n=32(\%)$ & $n=118(\%)$ & $n=150(\%)$ \\
\hline & CC & $12(37.5)$ & $53(44.9)$ & $61(43.2)$ \\
\hline & CT & $14(43.8)$ & $14(11.9)$ & $65(43.3)$ \\
\hline TCF7L2 rs7903746 & TT & $6(18.8)$ & $n=236(\%)$ & $20(13.3)$ \\
\hline & & $n=64(\%)$ & $157(66.5)$ & $n=300(\%)$ \\
\hline & C & $38(59.4)$ & $79(33.5)$ & $195(65)$ \\
\hline
\end{tabular}

Note: Pearson's Chi-square test, $p=0.453$ (not significant); Fisher's exact test, $p=0.548$ (not significant), odds ratio (OR) $=1.07$ for CC vs. (TT and CT) in patients with periodontitis $95 \%$ confidence interval $(\mathrm{Cl}): 0.90-1.26$ and $\mathrm{OR}=0.79$ in nonperiodontitis patients $95 \% \mathrm{Cl}: 0.41-1.49$ ).

\section{Discussion}

Aim of the present study was to investigate the possible correlation of the TCF7L2 SNP rs7903146 with prediabetes/diabetes and periodontal condition in patients of Greek origin who fulfilled criteria of high risk for developing T2DM. In this patient sample, $20.7 \%$ displayed $\mathrm{HbA} 1 \mathrm{c} \geq 5.7$, indicating prediabetes/diabetes and were referred for further diagnostic procedures by their physicians. The present study did not reveal statistically significant difference regarding genotype frequencies for rs7903146 variants (C/T) in the TGF7L2 gene and either periodontal condition or presence of hyperglycemia (HbA1c levels $\geq 5.7$ ), but it was confirmed that the presence of HbA1c levels $>5.7$ affected clinical parameters of periodontal disease.

These data enhance the recent joint guidelines by the International Diabetes Federation and the European Federation of Periodontology which encourage the participation of dental practitioners into early diagnosis of prediabetes/diabetes. $^{2}$

T2DM and periodontal disease are complex disorders and can be the result of both genetic and environmental interactions. However, having a susceptible genetic variant does not necessarily imply that the disease will be developed unless environmental cues are present. SNPs serve as the basis of complex diseases' genetic variations, when identified, thus providing a means of predicting the possibility of development of metabolic abnormalities in individuals. ${ }^{19}$ The environmental susceptibility factors for both T2DM and periodontitis are well reported but hopefully the genetic side of these disease in preventive and management action plans will also be incorporated. ${ }^{20}$ Targeted genetic and environmental pharmacotherapy with possible personalized treatment depending on the genetic susceptibility of the individual is now at the cutting edge of both diabetes and periodontitis diagnosis.

The TCF7L2 gene polymorphisms is one of the most common loci associated with T2DM. ${ }^{21}$ Since the association between the TCF7L2 SNP rs7903146 and the risk of T2DM was first reported in a Genome Wide Association Study (GWAS) by Grant et $\mathrm{al}^{22}$ numerous studies with the same approach $^{21}$ ) and case control studies replicated this association. The rs7903146 T-allele has been positively associated with risk for T2DM in European, ${ }^{23}$ Indian, Thai, ${ }^{24}$ and Iranian populations, ${ }^{25}$ East Asian populations, namely, Japanese ${ }^{26}$ and Chinese, ${ }^{27}$ Turks, $^{28}$ Arabic, and Algerian, ${ }^{29}$ African, ${ }^{30}$ Brazilian, ${ }^{31}$ and African American populations. ${ }^{32}$

However, conflicting results have been reported in some studies in populations from China, Indonesia, ${ }^{33}$ Saudi Arabia, and the United Arab Emirates, ${ }^{34}$ Egypt, ${ }^{35}$ as well as in a Native American population of North America, African Americans, Brazilian, ${ }^{18}$ and Afro Caribbeans ${ }^{23}$ So far, the data suggest that the allelic frequencies of the rs7903146 SNP and possibly, the effect size of this SNP are variable worldwide. ${ }^{14}$

Regarding the Greek population, apart from a study by Katsoulis et $\mathrm{al}^{36}$ in which the role of TCF7L2 variants in the 
development of impaired glucose metabolism (including impaired fasting glucose [IFG] or T2DM) in patients with metabolic syndrome (MS) was investigated, the present study is the firstreportofanassociation between TCF7L2 rs7903146variants and prediabetes/T2DM. Christopoulos et $\mathrm{al}^{37}$ investigated the role of the rs7903146 variant in women with polycystic ovary syndrome, while Pappa et $\mathrm{al}^{38}$ and Potasso et $\mathrm{al}^{39}$ examined the association with gestational diabetes mellitus (GDM). Furthermore, a study by Boutsouris et al examined the allelic frequencies in T2DM patients and in a Greek population sample, respectively.

In the present study, the participants were separated into two subgroups based on their HbA1c levels. One subgroup comprised of patients exhibiting normal HbA1c values $(\mathrm{HbA} 1 \mathrm{c}<5.7 \%)$ while the other subgroup consisted of patients identified with hyperglycemia (HbA1c $\geq 5.7 \%$ ). The allelic and genotype frequencies for the TCF7L2 SNP rs7903146 did not exhibit a significant difference between the HbA1c > 5.7 and HbA1c < 5.7 groups, and they were not significantly associated with $\mathrm{HbA1c}$ levels $(\mathrm{OR}=0.95$; $95 \%$ : $0.80-1.12$ in patients with $\mathrm{HbA1c}<5.7$ and 1.23 95\% CI: 0.66 -2.29in patients with ${ }^{3} 5.7$ HbA1c). The minor T-allele frequency was slightly higher in the HbA1c $<5.7$ group (HbA1c $>5.7=32.3 \%$, HbA1c $<5.7=35.7 \%$ ), while the TT genotype frequency was similar in both groups (HbA1c $>5.7=12.9 \%$, HbA1c $<5.7=13.4 \%$ ). These results could be due to the lack of statistical power because of the low prevalence of patients with hyperglycemia as identified in the present study or due to the variety between ethnicities and populations.

The frequency of the risk allele in the control group was similar to the frequency reported by Boutsouris et $\mathrm{al}^{40}$ in a Greek population, as well as in Spanish, and Indian populations, ${ }^{41}$ but it was in general higher than the frequencies described in Europe (19.7-33.5\%). Furthermore, the frequency was higher compared to East Asian populations, where it is being represented at less than $5 \%$ and lower compared to Arabic and North African populations (24-44\%). ${ }^{34}$ The frequency of the risk allele in the $\mathrm{HbA} 1 \mathrm{c}>5.7$ group was similar to the frequency described in women with a history of GDM in Greece and in other Caucasian and Indian ${ }^{23}$ population studies, while it was higher compared to the frequency in East Asian populations where the T-allele was found to be usually occurring at levels less than $6 \%$. The T-allele frequency was also lower than the levels reported in Turkish, ${ }^{28}$ Arabic, Algerian (38-52\%), ${ }^{34}$ Brazilian (28-47,4\%), ${ }^{31}$ and African populations (36-46\%) but was close to United Kingdom African Caribbean ${ }^{23}$ and African American populations.

No statistical differences were observed in the present study regarding both this specific allelic and genotype distribution between patients with periodontitis and periodontally healthy individuals as shown in - Table $\mathbf{3}$ (Pearson's Chi-square test, $p=0.453$ [not significant], Fisher's exact test, $p=0.548$ [not significant]). To our knowledge, this is the first report in the literature, reporting on the possible relation of this specific SNP with the presence of periodontitis and/or the levels of HbA1c. Further studies, with larger patient samples are required in order to investigate the above described genetic approach of interaction of these two diseases.
In agreement with a number of studies in the literature, the present study confirmed the relationship of hyperglycemia with inflammation of the periodontal tissues, since as reported in the "Results" section, clinical parameters of periodontal disease, such as probing depth, clinical attachment level and bleeding on probing, differed statistically significantly between patients with $\mathrm{HbA} 1 \mathrm{c}<5.7$ and those with HbA1c $\geq 5.7$ (Mann-Whitney $U$-test, $p<0.05$ ).

\section{Conclusion}

In summary, the present study did not reveal statistically significant difference regarding genotype frequencies for rs7903146 variants (C/T) in the TGF7L2 gene and both periodontal condition and presence of hyperglycemia (HbA1c levels $\geq 5.7$ ). The presence of HbA1c levels $>5.7$ affected clinical parameters of periodontal disease.

\section{Funding \\ The study was partially funded by Pierre-Fabre Hellas (94032 ELKE, A.P.Th.).}

Conflict of Interest

None declared.

\section{References}

1 Papapanou PN, Sanz M, Buduneli N, et al. Periodontitis: consensus report of workgroup 2 of the 2017 World Workshop on the Classification of Periodontal and Peri-Implant Diseases and Conditions. J Periodontol 2018;89(suppl 1) :S173-S182

2 Sanz M, Ceriello A, Buysschaert M, et al. Scientific evidence on the links between periodontal diseases and diabetes: Consensus report and guidelines of the joint workshop on periodontal diseases and diabetes by the International Diabetes Federation and the European Federation of Periodontology. J Clin Periodontol 2018;45(2):138-149

3 Grigoriadis A, Sorsa T, Räisänen I, Pärnänen P, Tervahartiala T, Sakellari D. Prediabetes/diabetes can be screened at the dental office by a low-cost and fast chair-side/point-of-care aMMP-8 immunotest. Diagnostics (Basel) 2019;9(4):151

4 Mataftsi M, Koukos G, Sakellari D. Prevalence of undiagnosed diabetes and pre-diabetes in chronic periodontitis patients assessed by an HbA1c chairside screening protocol. Clin Oral Investig 2019;23(12):4365-4370

5 Isola G, Alibrandi A, Currò M, et al. Evaluation of salivary and serum ADMA levels in patients with periodontal and cardiovascular disease as subclinical marker of cardiovascular risk. J Periodontol 2020

6 Isola G, Polizzi A, Alibrandi A, Williams RC, Leonardi R. Independent impact of periodontitis and cardiovascular disease on elevated soluble urokinase-type plasminogen activator receptor (suPAR) levels. J Periodontol 2020

7 Isola G, Polizzi A, Patini R, Ferlito S, Alibrandi A, Palazzo G. Association among serum and salivary A. actinomycetemcomitans specific immunoglobulin antibodies and periodontitis. BMC Oral Health 2020;20(1):283

8 Gupta N, Gupta ND, Gupta A, Goyal L, Garg S. The influence of type 2 diabetes mellitus on salivary matrix metalloproteinase- 8 levels and periodontal parameters: a study in an Indian population. Eur J Dent 2015;9(3):319-323

9 Kolb H, Martin S. Environmental/lifestyle factors in the pathogenesis and prevention of type 2 diabetes. BMC Med 2017;15(1):131 
10 Gaulton KJ. Mechanisms of type 2 diabetes risk loci. Curr Diab Rep 2017;17(9):72

11 Fuchsberger C, Flannick J, Teslovich TM, et al. The genetic architecture of type 2 diabetes. Nature 2016;536(7614):41-47

$12 \mathrm{Xi} \mathrm{X}, \mathrm{Ma} \mathrm{J}$. A meta-analysis on genetic associations between Transcription Factor 7 Like 2 polymorphisms and type 2 diabetes mellitus. Genomics 2020;112(2):1192-1196

13 Ferreira MC, da Silva MER, Fukui RT, Arruda-Marques MDC, Dos Santos RF. TCF7L2correlation in both insulin secretion and postprandial insulin sensitivity. Diabetol Metab Syndr 2018;10:37

14 Lou L, Wang J, Wang J. Genetic associations between Transcription Factor 7 Like 2 rs7903146 polymorphism and type 2 diabetes mellitus: a meta-analysis of 115,809 subjects. Diabetol Metab Syndr 2019;11:56

15 Centers for Disease Control and Prevention (CDC). Diabetes report card 2017. Available at: https://www.cdc.gov/diabetes/pdfs/library/diabetesreportcard2017-508.pdf. Accessed February 4, 2021

16 Beagley J, Guariguata L, Weil C, Motala AA. Global estimates of undiagnosed diabetes in adults. Diabetes Res Clin Pract 2014;103(2):150-160

17 Zhang T, Yungang P, Zhou H, et al. The application of IFCC reference methods in methodological evaluation of glycated hemoglobin measurement systems. Chinese J Lab Med 2018;41:821-826

18 Barros CM, Araujo-Neto AP, Lopes TR, et al. Association of the rs7903146 and rs12255372 polymorphisms in the TCF7L2 gene with type 2 diabetes in a population from northeastern Brazil. Genet Mol Res 2014;13(3):7889-7898

19 Speed D, Cai N, Johnson MR, Nejentsev S, Balding DJ; UCLEB Consortium. Reevaluation of SNP heritability in complex human traits. Nat Genet 2017;49(7):986-992

20 Graves DT, Ding Z, Yang Y. The impact of diabetes on periodontal diseases. Periodontol 2000 2020;82(1):214-224

21 Saxena R, Gianniny L, Burtt NP, etal. Common single nucleotide polymorphisms in TCF7L2 are reproducibly associated with type 2 diabetes and reduce the insulin response to glucose in nondiabetic individuals. Diabetes 2006;55(10):2890-2895

22 Grant SF, Thorleifsson G, Reynisdottir I, et al. Variant of transcription factor 7-like 2 (TCF7L2) gene confers risk of type 2 diabetes. Nat Genet 2006;38(3):320-323

23 Humphries SE, Gable D, Cooper JA, et al. Common variants in the TCF7L2 gene and predisposition to type 2 diabetes in UK European Whites, Indian Asians and Afro-Caribbean men and women. J Mol Med (Berl) 2006;84(12):1005-1014

24 Plengvidhya N, Chanprasert C, Chongjaroen N, Yenchitsomanus PT, Homsanit M, Tangjittipokin W. Impact of KCNQ1, CDKN2A/2B, CDKAL1, HHEX, MTNR1B, SLC30A8, TCF7L2, and UBE2E2 on risk of developing type 2 diabetes in Thai population. BMC Med Genet 2018;19(1):93

25 Kalantari S, Sharafshah A, Keshavarz P, Davoudi A, Habibipour R. Single and multi-locus association study ofTCF7L2gene variants with susceptibility to type 2 diabetes mellitus in an Iranian population. Gene 2019;696:88-94

26 Kunika K, Tanahashi T, Numata S, et al. Common coding variant in the TCF7L2 gene and study of the association with type 2 diabetes in Japanese subjects. J Hum Genet 2008;53(11,12):972-982

27 Zheng X-Y, Ren W, Zhang S-H, et al. Correlation between single nucleotide polymorphisms of rs7903146 and rs11196218 atTCF7L2gene and the early phase insulin secretion of newly diagnosed patients with type 2 diabetes. Med J Chin P Lib Army 2011;36:269-272
28 Demirsoy IH, Aras N, Cinkir U. TCF7L2 rs7903146 gene variation is associated with risk of type 2 diabetes in Turkish population. J Clin Med Gen 2016;4:1-3

29 Ouhaibi-Djellouli H, Mediene-Benchekor S, Lardjam-Hetraf SA, et al. The TCF7L2 rs7903146 polymorphism, dietary intakes and type 2 diabetes risk in an Algerian population. BMC Genet 2014;15:134

30 Danquah I, Othmer T, Frank LK, Bedu-Addo G, Schulze MB, Mockenhaupt FP. The TCF7L2 rs7903146 (T) allele is associated with type 2 diabetes in urban Ghana: a hospital-based case-control study. BMC Med Genet 2013;14:96

31 Marquezine GF, Pereira AC, Sousa AG, Mill JG, Hueb WA, Krieger JE. TCF7L2 variant genotypes and type 2 diabetes risk in Brazil: significant association, but not a significant tool for risk stratification in the general population. BMC Med Genet 2008;9:106

32 Yan Y, North KE, Ballantyne CM, et al. Transcription factor 7-like 2 (TCF7L2) polymorphism and context-specific risk of type 2 diabetes in African American and Caucasian adults: the atherosclerosis risk in communities study. Diabetes 2009;58(1):285-289

33 Oktavianthi S, Saraswati MR, Suastika K, et al. Transcription factor 7-like 2 single nucleotide polymorphisms are associated with lipid profile in the Balinese. Mol Biol Rep 2018;45(5):1135-1143

34 Saadi H, Nagelkerke N, Carruthers SG, et al. Association of TCF7L2 polymorphism with diabetes mellitus, metabolic syndrome, and markers of beta cell function and insulin resistance in a population-based sample of Emirati subjects. Diabetes Res Clin Pract 2008;80(3):392-398

35 Mandour I, Darwish R, Fayez R, Naguib M, El-Sayegh S. TCF7L2gene polymorphisms and susceptibility to type 2 diabetes mellitus, a pilot study. Biomed Pharmacol J 2018;11:1043-1049

36 Katsoulis K, Paschou SA, Hatzi E, Tigas S, Georgiou I, Tsatsoulis A. TCF7L2 gene variants predispose to the development of type 2 diabetes mellitus among individuals with metabolic syndrome. Hormones (Athens) 2018;17(3):359-365

37 Christopoulos P, Mastorakos G, Gazouli M, et al. Genetic variants in TCF7L2 and KCNJ11 genes in a Greek population with polycystic ovary syndrome. Gynecol Endocrinol 2008;24(9):486-490

38 Pappa KI, Gazouli M, Economou K, et al. Gestational diabetes mellitus shares polymorphisms of genes associated with insulin resistance and type 2 diabetes in the Greek population. Gynecol Endocrinol 2011;27(4):267-272

39 Potasso L, Perakakis N, Lamprinou A, et al. Clinical impact of the TCF7L2 gene rs7903146 type 2 diabetes mellitus risk polymorphism in women with gestational diabetes mellitus: impaired glycemic control and increased need of insulin therapy. Exp Clin Endocrinol Diabetes 2020;128(10):663-666

40 Boutsouris K, Poumpouridou N, Katsarou M, et al. Frequency distribution of TCF7L2, MTNR1B, CDKAL1, SLC30A8 and FTO gene polymorphisms prediposing to type II diabetes mellitus and/or obesity in a greek population and their impact in personalized medicine.Arch Balk Med Union2016;51:157 -163

41 Gupta V, Khadgawat R, Ng HKT, et al. A validation study of type 2 diabetes-related variants of the tcf 72 , hhex, kcnj11, and adipoq genes in one endogamous ethnic group of north India. Ann Hum Genet 2010;74(4):361-368 